\title{
INTERFACE ENERGY SPECTRUM OF REAL PbTe/SnTe HETEROJUNCTION
}

\author{
V.I. LITVINOV \\ Institute of Material Science Problems, Ukr. Acad. Sci. \\ Krasnoarmeyskaya 60-63, 274029 Chernovtsy, Ukraine \\ AND M. OszWAEDoWSKI \\ Institute of Physics, Technical University of Poznań \\ Piotrowo 3, 60-965 Poznań, Poland
}

\begin{abstract}
The interface energy spectrum in real band-inverted $\mathrm{PbTe} / \mathrm{SnTe}$ heterojunctions formed both in the (111) and (001) planes is calculated. It is shown that even if the valence band of SnTe lies above the conduction band minimum of $\mathrm{PbTe}$, the interface, midgap states may still exist due to the strain effect on the band gaps.
\end{abstract}

PACS numbers: 73.40.Lq, 71.50.+t

\section{Introduction}

In heterojunctions formed by semiconductors having inverted order of the bands and overlapping gaps, there were found bands of electron and hole interface states which are gapless and have a linear dispersion [1,2]. A model of such a heterostructure is a $\mathrm{PbTe} / \mathrm{SnTe}$ heterojunction formed in the (111) plane. So far, all the results $[1,2]$ were limited to a single [111] valley which is directed perpendicularly to the junction. In the present contribution, the interface states associated with the remaining three [11i]-type valleys and the interface states generated when the junction is formed in a (001) plane are considered. The effect of the strain at the heterojunction is also considered.

\section{The Hamiltonian}

In the two-band model of a $\mathrm{A}_{>}^{4} \mathrm{~B}^{6}$ semiconductor the Hamiltonian is of the Dirac form

$$
H=\frac{E_{\mathrm{g}}}{2} \beta+\sum_{i, j} \alpha_{i} v_{i j} p_{j}, \quad v_{i j}=\left(\begin{array}{ccc}
v_{\perp} & 0 & 0 \\
0 & v_{\perp} & 0 \\
0 & 0 & v_{z}
\end{array}\right),
$$




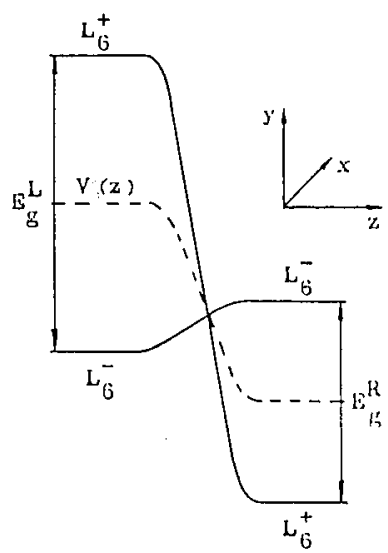

Fig. 1. Diagram of the inverted-band heterojunction.

where $p=-\mathrm{i} \hbar \nabla, \alpha_{i}$ and $\beta$ are the Dirac matrices, $v_{z}$ and $v_{\perp}$ are interband matrix elements having the dimension of velocity, and $i, j=x, y, z$. The $z$ axis is chosen to be parallel to the (111) direction.

In order to determine the energy states at the interface, it is assumed that the junction is formed at $z=0$ (Fig. 1) and that the energy gap depends on $z\left(E_{\mathrm{g}}=E_{\mathrm{g}}(z)\right)$ in such a way that for $z=\infty, E_{\mathrm{g}}(\infty)=E_{\mathrm{g}}^{\mathrm{R}}>0$, and for $z=-\infty, E_{\mathrm{g}}(-\infty)=E_{\mathrm{g}}^{\mathrm{L}}<0$.

The alignment of the gap centers is described by a function $V(z)$, which for an abrupt junction is of the form [2]:

$$
V(z)=V_{0}+\frac{\lambda}{2} E_{\mathrm{g}}(z)
$$

where

$$
V_{0}=V^{\mathrm{L}}-\frac{\lambda}{2} E_{\mathrm{g}}^{\mathrm{L}}=V^{\mathrm{R}}-\frac{\lambda}{2} E_{\mathrm{g}}^{\mathrm{R}}
$$

is the value of $V(z)$ at the crossover point, while $V^{\mathrm{L}}$ and $V^{\mathrm{R}}$ are the values of $V(z)$ for $z=-\infty$ and $z=+\infty$ respectively. The parameter $\lambda$ is defined as

$$
\lambda=\frac{2\left(V^{\mathrm{L}}-V^{\mathrm{R}}\right)}{E_{\mathrm{g}}^{\mathrm{L}}-E_{\mathrm{g}}^{\mathrm{R}}} .
$$

If the gaps are aligned $(V(z)=$ const); the wave functions which describe bound states at the interface are of the form

$$
\Psi_{ \pm}=\left(\begin{array}{r} 
\pm \exp (\mathrm{i} \Theta / 2) \\
\mathrm{i} \exp (\mathrm{i} \Theta / 2) \\
\pm \mathrm{i} \exp (-\mathrm{i} \Theta / 2) \\
\exp (\mathrm{i} \Theta / 2)
\end{array}\right) \exp (\mathrm{i} k \cdot r) \exp \left[\left(-1 / 2 \hbar v_{z}\right) \int_{0}^{z} E_{\mathrm{g}}(z) \mathrm{d} z\right]
$$

where $\exp (\mathrm{i} \Theta)=\left(p_{x}+\mathrm{i} p_{y}\right) / p, p=\sqrt{p_{x}^{2}+p_{y}^{2}}$. The scale of variation of the wave functions is $l=E_{\mathrm{g}}(\infty) / 2 \hbar v_{z}$. 


\section{The interface states}

Solving the eigenvalue problem at the condition of Eq. (2), one obtains the following gapless interface spectra:

- Junction in (111) plane, the [111] valley

$$
E_{ \pm}=V_{0} \pm v_{\perp} p \sqrt{1-\lambda^{2}}
$$

with "+" for electrons and "-" for holes.

- Junction in (111) plane, the (11ī]-type valleys These valleys make an angle $\Theta$ with the $z$ direction such that $\cos \theta=1 / 3$. Transforming the Hamiltonian for these valleys to the junction coordinate system one obtains

$$
E_{ \pm}=V_{0} \pm \frac{5 v_{\perp}+4 v_{z}}{9} p \sqrt{1-\lambda^{2}}
$$

This threefold degenerate branch coexists with that of Eq. (4).

- Junction in (001) plane

In this case there are four equivalent [111] valleys which make an angle $\Theta$ with the $z$ axis, such that $\cos \theta=1 / \sqrt{3}$. Performing relevant Hamiltonian transformation one obtains fourfold degenerate spectrum

$$
E_{ \pm}=V_{0} \pm \frac{v_{\perp}+2 v_{z}}{9} p \sqrt{1-\lambda^{2}}
$$

The interface spectra of Eqs. (4)-(6) exist only if the energy gaps at least partly overlap.

\section{The effect of strain}

Owing to the difference in the lattice constants of $\mathrm{PbTe}, a=6.45 \AA$, and of SnTe, $a=6.31 \AA[3]$, the $\mathrm{PbTe} / \mathrm{SnTe}$ heterojunction is strained. The resulting in-plane strain, $\varepsilon=2 \times 10^{-2}$, is compressive for $\mathrm{PbTe}$ and expansive for $\mathrm{SnTe}$. The effect of the strain is to shift the valence and the conduction bands. The shifts depend on the deformation potentials of the bands and on the elastic stiffness coefficients of the junction materials [4]. In the present calculations the elastic coefficients wcre taken from Ref. [3], and the deformation potentials from the calculations of Rabii [5] for SnTe and of Fereira [6] for PbTe. The data of Rabii and Fereira were divided by a factor of 1.09 , to fit them to the energy gap hydrostatic pressure coefficients of $-7 \times 10^{-11} \mathrm{eV} / \mathrm{Pa}$ for SnTe and $+8.8 \times 10^{-11} \mathrm{eV} / \mathrm{Pa}$ for $\mathrm{PbTe}$ [3].

Results of the energy shifts calculations are shown in Fig. 2. The band offset $\Delta$ seen in Fig. 2 is not known. It is however found $[7,8]$ that $\Delta>0$. In this case, it can be concluded from Fig. 2 that the interface states can exist only if in the (111) junction PbTe is strained or if in the (001) junction SnTe is strained. The strained energy gaps enter Eq. (2) only if the localization length $l$ of the interface excitations in $z$ direction is smaller than the critical thickness $h$ for the generation of the misfit dislocations. Otherwise, for $L>h$ the energy gaps which enter Eq. (2) are the bulk gaps, which means that the strain does not affect the interface energy 

a. Heterojunction in (111)
b. Heterojunction in (001)

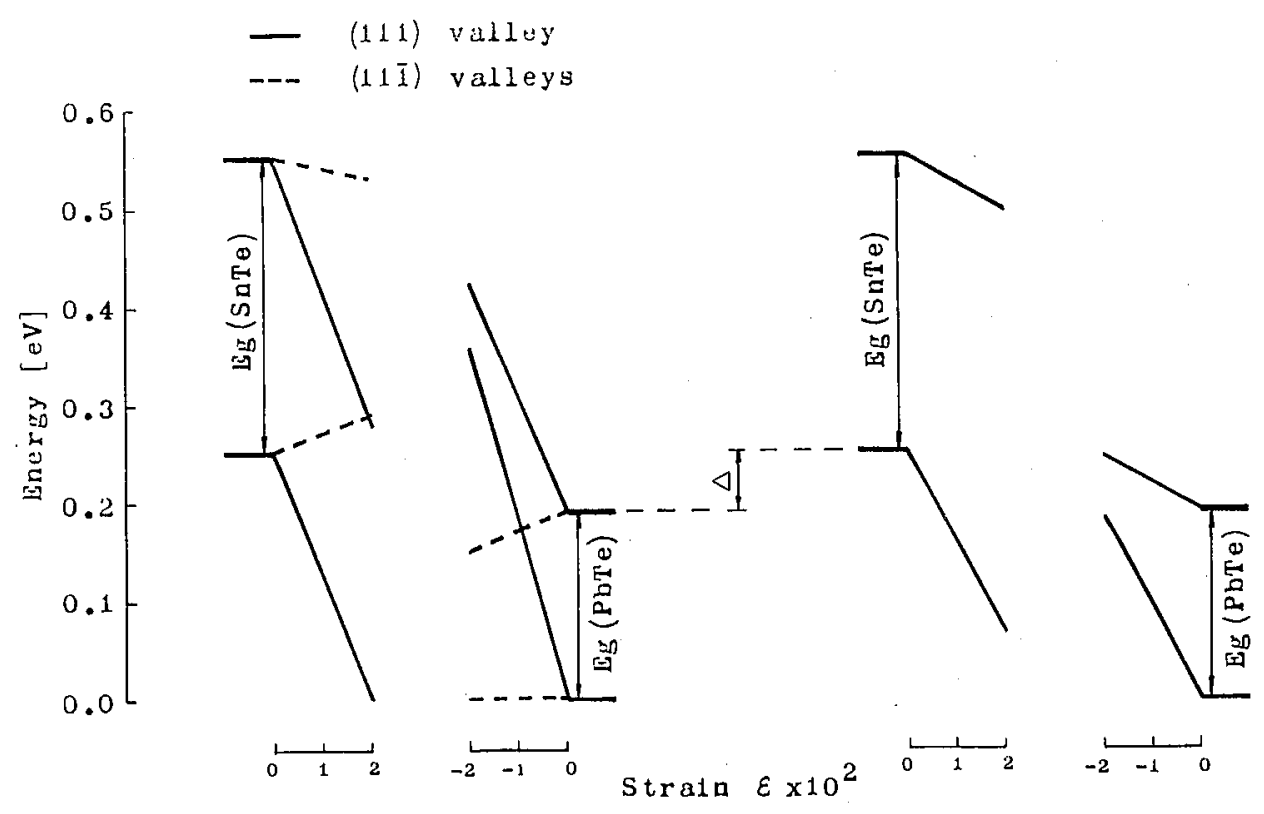

Fig. 2. The strain effect on the energy gaps of SnTe and PbTe.

spectrum. The estimation for the materials in question give $10 \AA<l<40 \AA$, and $h \approx 100 \AA[7]$. Thus, if in $\mathrm{PbTe} / \mathrm{SnTe}$ junction $\Delta>0$, the interface excitations can exist only due to the strain effects.

\section{References}

[1] B.A. Volkov, O.A. Pankratov, JETP Lett. 42, 178 (1985).

[2] D. Agassi, V. Korenman, Phys. Rev. B 37, 10095 (1988).

[3] G. Nimtz, B. Schlicht, R. Dornhaus, Narrow-Gap Semiconductors, Springer Tracts in Modern Physics, Springer, Berlin 1983.

[4] J. Singleton, E. Kress-Rogers, A.V. Lewis, R.J. Nicholas, E.J. Fantner, G. Bauer, A. Otero, J. Phys. C 19, 77 (1986).

[5] S. Rabii, Phys. Rev. 182, 821 (1969).

[6] L.G. Fereira, Phys. Rev. 137, A1601 (1965).

[7] I.G. Neizvestny, A.M. Palkin, V.N. Shumski, V.N. Sozinov, E.T. Stakevich, O.I. Vasin, Superlattices Microstruct. 3, 291 (1991).

[8] S. Takaoka, T. Okumura, K. Murase, A. Ishida, H. Fujiyasu, Solid State Commun. 58, 637 (1986).

[9] A.I. Fedorenko, O.N. Nashchekina, B.A. Savitskii, L.P. Shpakovskaya, O.A. Mironov, M. Oszwałdowski, Vacuum, in press. 